\title{
THE TREATMENT OF ISOLATED FRACTURES OF THE DISTAL ULNA
}

\author{
I. W. D. DYMOND
}

From the Baragwanath, J. G. Strydom and Hillbrow Hospitals, Johannesburg

\begin{abstract}
The integrity of the periosteum and the interosseous membrane determine the stability of fractures of the distal ulna; this is indicated by the initial displacement. In fractures displaced by less than $50 \%$ the periosteum and interosseous membranes are largely intact; these fractures are stable and require only belowelbow immobilisation for protection and relief of pain. In fractures displaced by more than $50 \%$ the membranes are disrupted; these fractures are unstable and require above-elbow immobilisation for stability. As most fractures are displaced by less than $50 \%$, immobilisation of the elbow, which significantly increases morbidity, is usually unnecessary. I report the results of a cadaveric study on the pathomechanics of fractures of the distal ulna, and of a prospective clinical trial in which the type of cast used for immobilisation was determined by the stability of the fracture.
\end{abstract}

Isolated fractures of the distal ulna are usually caused by direct trauma, as when the forearm is used to block an assault to the head and neck (hence the term "night-stick fracture"). In South Africa, where "knobkerries" (wooden sticks with heavy rounded ends) and other blunt objects are used as weapons, the injury is common.

The management of these fractures seems to be simple, yet there is considerable variation. Immobilisation in an above-elbow cast is probably the most frequently used treatment for undisplaced fractures (Watson-Jones 1952), and open reduction with internal fixation for displaced fractures (Anderson 1975). Unorthodox methods such as simple bandaging are also known to be used with apparently no adverse results. Modification of the treatment by the patient himself is also not uncommon; for example, one of our patients kept only the above-elbow portion of his cast after the first week. Despite these variations in management the fractures unite.

In our hospital most ulnar fractures are treated by immobilisation in a plaster cast, extending distally to the knuckles and proximally either to above the elbow or below it, the choice being somewhat random. But which is best? To answer this question I studied the pathomechanics of the fracture in cadavers. Based on this study two types of fracture were identified and, in a prospective clinical trial, treated differently. The results of both the cadaveric study and the clinical trial are reported here.

I. W. D. Dymond, MB BCh, FRCSEd, FCS (SA)Orth, Registrar Department of Orthopaedic Surgery, University of the Witwatersrand Medical School, York Road. Parktown, 2193 Johannesburg, South Africa.

(C) 1984 British Editorial Society of Bone and Joint Surgery $0301-620 \mathrm{X} / 84 / 3043 \$ 2.00$

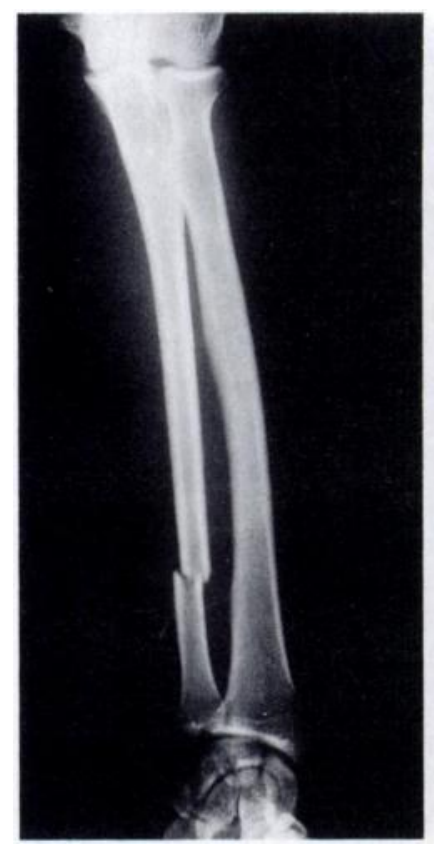

Fig. 1

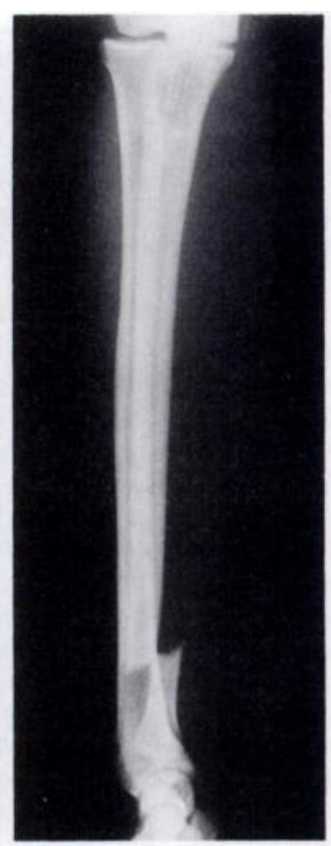

Fig. 2
Figure 1-Type I fracture: there is less than $50 \%$ displacement; this indicates minimal loss of soft-tissue integrity. Figure $2-$ Type II fracture: there is more than $50 \%$ displacement; this indicates disruption of the interosseous membranes.

\section{EXPERIMENTAL STUDY}

Ten fresh cadavers were used to study the pathomechanics of fractures of the distal ulna. At first, a fracture model produced by osteotomising the distal third of the ulna with a Gigli's saw was used, leaving the periosteum and the interosseous membrane intact. The bone across the osteotomy site was marked with a reference line and the forearm put through a full range of movement. Rotation, angulation and displacement of the distal fragment were minimal. The periosteum and the inter- 
osseous membrane were then divided for $2 \mathrm{~cm}$ on either side of the osteotomy and the movements of the forearm repeated. Displacement of the distal fragment of the ulna was now possible; rotation and angulation occurred with pronation and supination.

Next, on different cadavers, the distal ulna was fractured by a blow with a blunt instrument, and the specimens dissected. It was apparent that when the fracture was displaced by less than $50 \%$ the interosseous membrane was largely intact and the periosteum was minimally disrupted; these fractures were stable through a full range of movement. When the fracture was displaced by more than $50 \%$, marked periosteal tearing and separation of the interosseous membrane occurred; pronation and supination of the forearm showed that the fracture was unstable.

From these observations I classified fractures of the distal ulna into two types: Type I-fractures with less than $50 \%$ displacement, which are stable (Fig. 1); and Type II-fractures with more than $50 \%$ displacement, which are unstable (Fig. 2).

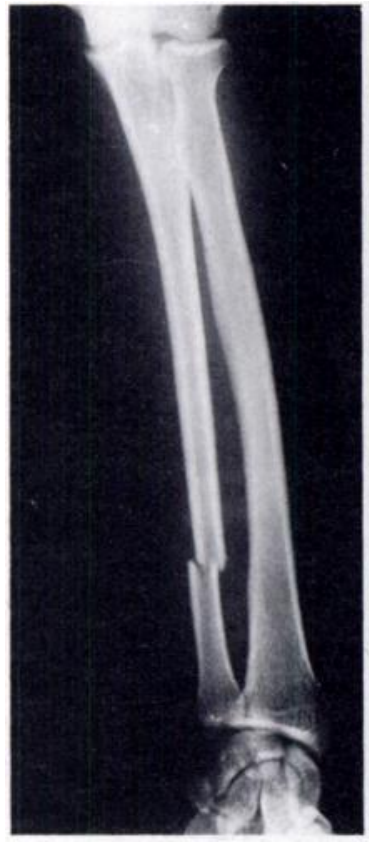

Fig. 3

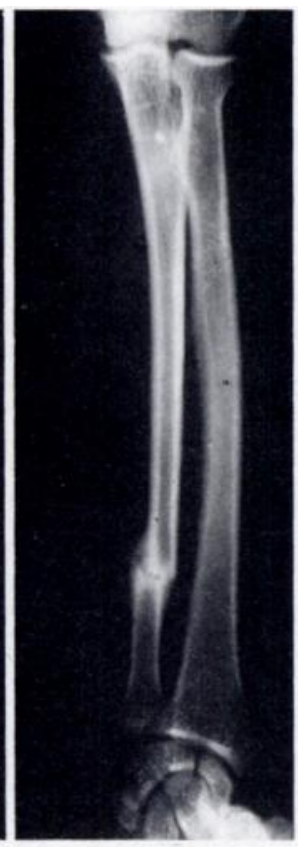

Fig. 4
Figure 3-Type I fracture before treatment in a belowelbow cast. Figure 4-After six weeks' immobilisation: the cast was used more for protection than for immobilisation.

\section{CLINICAL TRIAL}

A prospective clinical trial of 46 patients with isolated fractures of the distal ulna was conducted; treatment was determined by the classification described.

Thirty-nine patients with displacement of less than $50 \%$ (Type I fractures) were treated in a below-elbow cast for six weeks. During this time they continued their normal daily activities. When the cast was removed all had a full range of pronation and supination.

Seven patients with displacement of more than $50 \%$ (Type II fractures) were treated with above-elbow casts for six weeks. Three were unable to work during this period. After the casts were removed these patients took two to three weeks to regain full movement, and had considerable discomfort during this time.

All 46 patients showed clinical union after six weeks of immobilisation (Figs 3 and 4). Radiographic union was complete after nine weeks in those patients who were treated with a below-elbow cast, and after 12 weeks in those treated in an above-elbow cast.

Further displacement occurred in two fractures that had initially been displaced by less than $50 \%$; both were in the distal third of the ulna and both were comminuted. Union occurred with no delay or complications.

Radiographic study. Radiographs of six patients were taken before their fractures were immobilised. Under

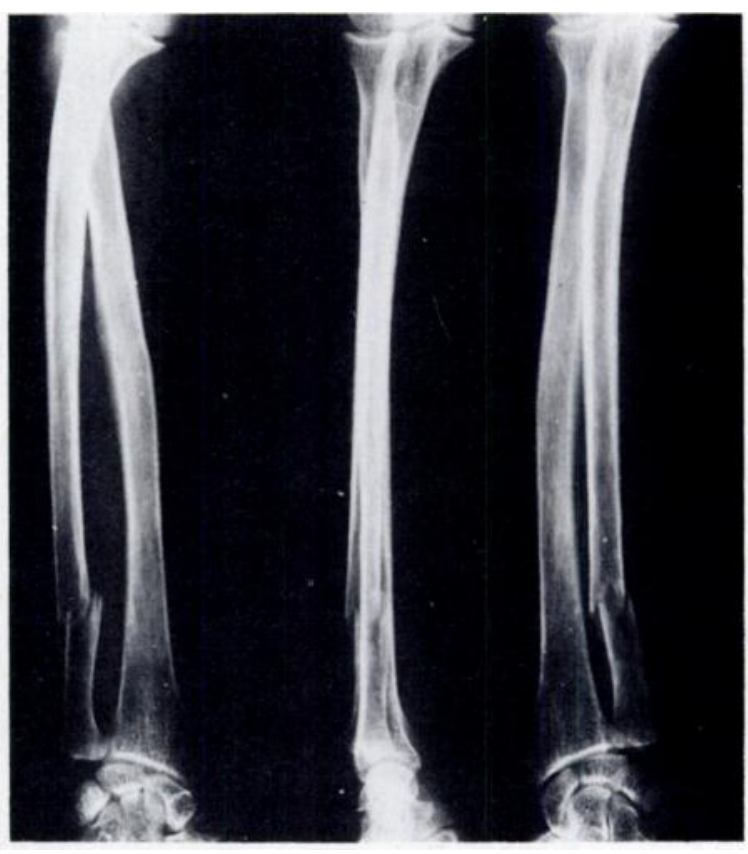

Fig. 5

Type I fracture in supination, neutral and pronation demonstrating stability of the ulna throughout the entire range.

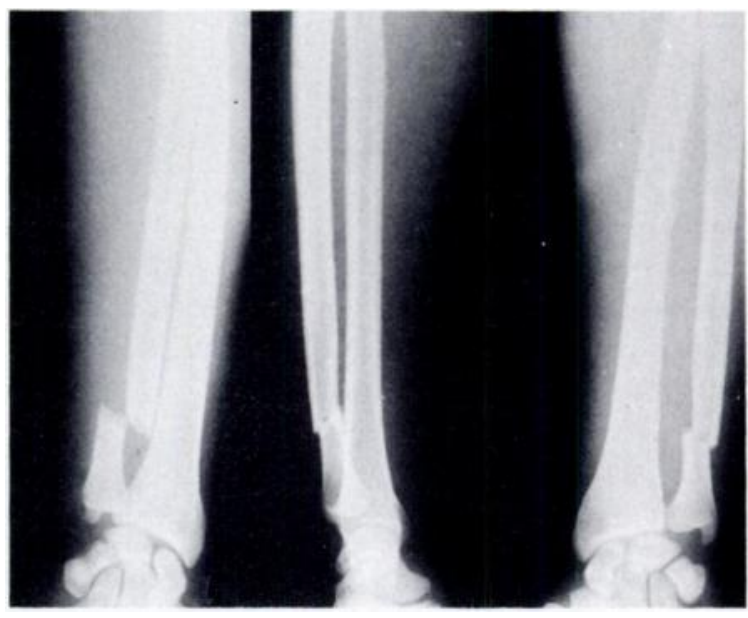

Fig. 6

Type II fracture in supination, neutral and pronation demonstrating instability of the ulna with movement. 
regional anaesthesia the injured forearm was rotated from pronation to supination and back again, observing the stability of the fracture: in Type I fractures no significant displacement occurred, whereas in the Type II fractures considerable movement at the fracture site took place (Figs 5 and 6).

\section{DISCUSSION}

The standard method of treatment for isolated fractures of the distal ulna is immobilisation in an above-elbow cast for six to eight weeks (Du Toit and Gräbe 1979). This method follows the principle that immobilisation of the joints above and below the fracture will prevent rotational forces acting on the fragments. But is this immobilisation of the forearm always necessary?

When pronation and supination of the forearm are considered, it is clear that only the radius rotates round the ulna; the ulna cannot rotate because its articulation with the trochlea of the humerus permits only flexion and extension. The radius, pivoting at the radiohumeral joint and hinging at the interosseous membrane and the distal radio-ulnar joint, swings around the ulna. The interosseous membrane not only unites the two forearm bones, but also acts as a restraint to rotational movement of the forearm. Thus, in a fracture of the distal ulna where the interosseous membrane is intact, little movement can occur in the distal fragment. We have shown that in fractures with less than $50 \%$ displacement, the interosseous membrane is largely intact. In this situation the periosteum which also is mostly intact, helps to stabilise the fracture.

Conversely, in fractures of the ulna where the interosseous membrane and the periosteum are disrupted, both these stabilising structures are lost; we found this in fractures with more than $50 \%$ displacement.

In conclusion, it is suggested that the stability of isolated fractures of the distal ulna is dependent on the integrity of the periosteum and interosseous membrane. This stability can be assessed on radiographs by the amount of initial displacement of the fragments : fractures with less than $50 \%$ displacement are stable and those with more than $50 \%$ are unstable.

In my experience, most fractures sustained while warding off a blow are Type I fractures and need only a below-elbow cast. This allows considerably more freedom for the activities of daily life than an above-elbow cast. To treat all fractures of the ulna with an above-elbow cast for the sake of the few unstable fractures which need it, is not justified.

I would like to thank Mr E. H. W. Erken for his help and encouragement with the editing of this paper and Mr S. Pincus, Mr A. Keays and Mr S. Eisenstein for allowing me to study their patients.

\section{REFERENCES}

Anderson LD. Fractures of the shafts of the radius and ulna. In: Rockwood CA, Green DP, eds. Fractures. Philadelphia and Toronto: JB Lippincott, 1975:1:441-85.

Du Toit FP, Gräbe RP. Isolated fractures of the shaft of the ulna. $S$ Afr Med J 1979:56:21-5.

Watson-Jones Sir R. Fractures and joint injuries. 4th ed. Edinburgh and London: E \& S Livingstone 1952:1. 